\title{
Metformin protects against oxidized low density lipoprotein-induced macrophage apoptosis and inhibits lipid uptake
}

\author{
NING HUANGFU, YONG WANG, JINGSONG CHENG, ZHENYU XU and SHENGHUANG WANG \\ Department of Cardiology, Ningbo First Hospital, Ningbo, Zhejiang 315010, P.R. China
}

Received July 11, 2017; Accepted December 28, 2017

DOI: $10.3892 /$ etm.2018.5704

\begin{abstract}
Oxidized low density lipoprotein (ox-LDL)-induced macrophage apoptosis contributes to the formation of atherosclerosis. Metformin, an antidiabetic drug, has been reported to attenuate lipid accumulation in macrophages. In this study, the effects of metformin on ox-LDL-induced macrophage apoptosis were investigated and the mechanisms involved in this process were examined. By performing flow cytometry analysis, it was demonstrated that metformin inhibited ox-LDL-induced macrophage apoptosis. Increased expression of endoplasmic reticulum (ER) stress marker proteins, including $\mathrm{C} / \mathrm{EBP}$-homologous protein, eukaryotic translation initiation factor $2 \mathrm{~A}$, and glucose-regulated protein $78 \mathrm{kDa}$, induced by ox-LDL was also reversed by metformin. Furthermore, ox-LDL-induced cytochrome $c$ (cyto-c) release and mitochondrial membrane potential loss were inhibited by metformin. As lipid uptake in macrophages contributed to ER stress, cyto-c release and mitochondrial membrane potential loss, the mechanisms involved in metformin-inhibited macrophage lipid uptake were investigated. Expression of scavenger receptors, including scavenger receptor A, cluster of differentiation 36 and lectin-type oxidized LDL receptor 1 was examined in the presence or absence of metformin with ox-LDL treatment. Additionally, the upstream regulatory mechanism of scavenger receptors by metformin was also analyzed. In conclusion, metformin protects against ox-LDL-induced macrophage apoptosis and inhibits macrophage lipid uptake.
\end{abstract}

\section{Introduction}

Atherosclerosis, which results in a significant number of deaths worldwide annually, is becoming a serious burden in

Correspondence to: Dr Shenghuang Wang, Department of Cardiology, Ningbo First Hospital, 59 Liuting Road, Ningbo, Zhejiang 315010, P.R. China

E-mail: 13605746913@163.com

Key words: metformin, macrophage, ox-LDL, apoptosis, lipid uptake almost all countries $(1,2)$. As a chronic immune-inflammatory disease, macrophages in the arterial wall engulf oxidized low density lipoprotein (ox-LDL) and undergo apoptosis, which contributes to the formation of foam cells. The assembly of foam cells develops into atherosclerotic plaque and accelerates atherosclerosis development $(3,4)$. During this process, ox-LDL-load macrophage apoptosis and lipid uptake play the key role. Relieving the ox-LDL-induced cholesterol metabolism obstacle in macrophages may play a vital role in alleviating atherosclerosis.

Metformin, a widely used anti-T2D (type 2 diabetes) drug (5), exerts anti-hyperglycemic effects by inhibiting gluconeogenesis in the liver, strengthening glucose uptake in muscles and suppressing glucose absorption from the small intestine $(6,7)$. Several researchers have reported that metformin can slow or reverse the progress of the atherosclerotic plaque in patients with diabetes and impaired glucose tolerance (IGT) (8). Moreover, metformin also inhibited lipid accumulation and cholesterol biosynthesis in macrophages $(9,10)$. However, the detailed effect of metformin on macrophages warrants further investigation.

In the present study, we further explored the function of metformin on ox-LDL-induced macrophage apoptosis and the possible mechanism, including endoplasmic reticulum (ER) stress, mitochondrial membrane potential depolarization, cytochrome $c$ (cyto-c) release, and lipid uptake.

\section{Materials and methods}

Cell culture. The THP-1 cell line was purchased from American Type Culture Collection (Manassas, VA, USA) and was maintained in RPMI-1640 culture medium containing $10 \% \mathrm{FBS}, 100 \mathrm{U} / \mathrm{ml}$ penicillin and $100 \mu \mathrm{g} / \mathrm{ml}$ streptomycin (all from Gibco; Thermo Fisher Scientific, Inc., Waltham, MA, USA). Cells were passaged approximately twice per week to maintain logarithmic growth and were cultured at $37^{\circ} \mathrm{C}$ and $5 \% \mathrm{CO}_{2}$ in a humidified incubator. Macrophages were obtained as described (11). Briefly, THP1 cells $\left(2 \times 10^{5}\right.$ cells $\left./ \mathrm{ml}\right)$ were cultured in a medium with $100 \mathrm{nM}$ phorbol-12-myristate 13-acetate (PMA; Sigma-Aldrich; Merck KGaA, Darmstadt, Germany) for 3 days. Next, the PMA-containing media was discarded, and cells were cultured in fresh RPMI-1640 (10\% FBS, $100 \mathrm{U} / \mathrm{ml}$ penicillin and $100 \mu \mathrm{g} / \mathrm{ml}$ streptomycin) for another $24 \mathrm{~h}$ to obtain macrophages for later experiments. 
Western blotting. Protein extraction and immunoblotting were performed as follows: In brief, cells were lysed, and the supernatant was collected. Concentrations of proteins was determined by the BCA assay kit (Beyotime Institute of Biotechnology, Shanghai, China). An equal amount of proteins $(40 \mu \mathrm{g})$ were resolved and run on a $10 \%$ polyacrylamide SDS gels and transferred onto polyvinylidenedifluoride membranes (EMD Millipore, Billerica, MA, USA). Immunoblotting was performed using relevant antibodies respectively. Immuno-detection was accomplished using a mouse anti-rabbit or goat anti-mouse secondary antibody and later visualized using ECL detection (Pierce; Thermo Fisher Scientific, Inc.). The GAPDH protein was used as endogenous control. Primary antibodies used were as follows: Rabbit anti-Bak antibody (1:1,000; Abcam, Cambridge, MA, USA); rabbit anti-Bax antibody (1:1,000; Abcam); rabbit; rabbit anti-Bad antibody (1:1,000; Abcam); rabbit anti-Bcl-2 antibody (1:1,000; Abcam); mouseanti-GAPDHantibody(1:2,000;Nuoyang,Beijing,China); rabbit anti-cleaved PARP antibody (1:1,000; Abcam); rabbit anti-glucose-regulated protein 78 (GRP78) antibody (1:1,000; Abcam); rabbit anti-PDI antibody (1:1,000; Abcam); rabbit anti-PERK antibody (1:1,000; Abcam); rabbit anti-CHOP antibody (1:1,000; Abcam); rabbit anti-p-eIFa antibody (1:1,000; Abcam); rabbit anti-CD36 antibody (1:1,000; Abcam); rabbit anti-SRA antibody (1:1,000; EMD Millipore); rabbit anti-LOX-1 antibody (1:1,000; Abcam); rabbit anti- $\beta$-catenin antibody $(1: 1,000$; Cell Signaling Technology, Inc., Danvers, MA, USA); rabbit anti-PPAR- $\gamma$ antibody (1:1,000; Cell Signaling Technology, Inc.); rabbit anti-AP-1 antibody (1:1,000; Abcam); rabbit anti-lamin B antibody (1:2,000; Abcam).

Nuclear protein isolation. Nuclear protein extraction was performed using the nuclear and cytoplasmic protein extraction kit (Beyotime Institute of Biotechnology,) according to the manufacturer's directions. In brief, cells were harvested and resuspended using buffer A on ice, mixed with buffer B and certrifugated. Cell debris was collected and later lysed using buffer $\mathrm{C}$ for $10 \mathrm{~min}$ on ice. After certrifugated, supernatant was collected as nuclear protein extraction. Western blots were performed as previous description with Lamin B used as a loading control for the analysis of nuclear protein expression, while GAPDH was used as a loading control for the analysis of total protein expression.

Flow cytometry analysis. The flow cytometry detection was performed using the FITC Annexin V Apoptosis Detection kit I (BD Biosciences, Franklin Lakes, NJ, USA) according to the manufacturer's directions. Briefly, the harvested cells were washed twice with cold PBS and later resuspended in $1 \mathrm{X}$ binding buffer at a concentration of $1 \times 10^{6}$ cells $/ \mathrm{ml}$. Next, $100 \mu \mathrm{l}$ of solution $\left(1 \times 10^{5}\right.$ cells $)$ were transferred to a culture tube, and $5 \mu \mathrm{l}$ of FITC Annexin V and $5 \mu \mathrm{l}$ of PI were added. Tubes were gently vortexed and incubated for $15 \mathrm{~min}$ at room temperature in the dark, and $400 \mu \mathrm{l}$ of $1 \mathrm{X}$ binding buffer was added to each tube. Cells were analyzed using CellQuest software (BD Biosciences).

Immunofluorescence staining. Immunofluorescence staining was performed as follows: First, cells were fixed in $4 \%$ paraformaldehyde for $30 \mathrm{~min}$ at room temperature. Then, cells were permeabilized with $0.2 \%$ Triton X-100 for $10 \mathrm{~min}$. After blocking in $2 \%$ BSA for $30 \mathrm{~min}$, cells were incubated with primary antibodies against CD36 and SRA overnight at $4^{\circ} \mathrm{C}$. Later, after incubating for $1 \mathrm{~h}$ at $37^{\circ} \mathrm{C}$ with the appropriate fluorescent-conjugated secondary antibody, cells were counterstained with 4',6-diamidino-2-phenylindole (DAPI; Roche Diagnostics, Basel, Switzerland) and examined with a fluorescence microscope (Nikon TE300; Nikon Corporation, Tokyo, Japan). All images were collected with the same settings for software and hardware.

Mitochondrial membrane potential (MMP) analysis. Mitochondrial membrane potential was measured by 5,5',6,6'Tetrachloro-1,1',3,3'tetraethylbenzimidazolylcarbocyanine iodide (JC-1; Sigma-Aldrich; Merck KGaA) staining. Cells were incubated with $2 \mu \mathrm{M} \mathrm{JC}-1$ at $37^{\circ} \mathrm{C}$ for $10 \mathrm{~min}$. After washing three times with PBS, cells were visualized using a fluorescence microscope (Axio Observer Z1, CCD camera; Carl Zeiss AG, Oberkochen, Germany).

Lipid uptake detection. Cells were washed with RPMI-1640 (Gibco; Thermo Fisher Scientific, Inc.) twice and incubated with dil-ox-LDL (10 $\mu \mathrm{g} / \mathrm{ml}$; Yiyuan Biotech Inc., Guangzhou, China) at $37^{\circ} \mathrm{C}$ for $30 \mathrm{~min}$ in the dark according to the manufacturer's instruction. Cells were washed with PBS for total of $15 \mathrm{~min}$ in the dark. Cells were observed and photos were taken using an Olympus fluorescence microscope (Olympus Corporation, Tokyo, Japan).

Statistical analysis. All the experiments were repeated three times. Data were presented as the mean \pm standard deviation. Statistically significant differences among three or more groups, statistical analysis was performed using one-way analysis of variance (ANOVA). $\mathrm{P}<0.05$ was considered to indicate a statistically significant difference.

\section{Results}

Metformin suppresses ox-LDL loaded-macrophage apoptosis. As reported by previous studies $(12,13)$, ox-LDL did not influence macrophage apoptosis alone. Combined with the ER stress activator-thapsigargin (TG), ox-LDL prominently induced macrophage apoptosis (Fig. 1A) $(14,15)$. When macrophages were treated with metformin excessively with the indicated concentrations $(0.1,0.3,0.5,1 \mathrm{mM})$ for $24 \mathrm{~h}$, the percentage of apoptotic cells was decreased from 16.9 to $6.0 \%$ (Fig. 1A). After pretreatment with metformin for $24 \mathrm{~h}$, macrophages were later stimulated with ox-LDL and TG. The proportion of apoptotic cells was also reduced (Fig. 1B). Next, PARP cleavage, triggered by caspase-3 activation during apoptotic processes, and apoptosis-related Bcl-2 family proteins were also detected when macrophages were treated with ox-LDL TG and metformin $(0.5 \mathrm{mM})$. As shown in Fig. 2A, there was no difference in the expression of full-length caspase-3 and PARP among these groups as expected. But, PARP cleavage and caspase- 3 activation were induced by ox-LDL and TG while metformin attenuated it. Levels of the pro-apoptotic proteins Bax and Bak were increased and the anti-apoptotic proteins $\mathrm{Bcl}-2$ and $\mathrm{Bad}$ were 

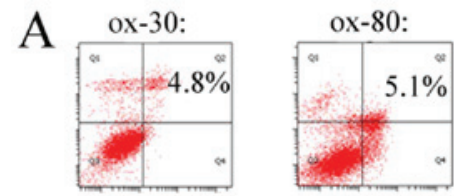

$\mathrm{ox}+\mathrm{TG}+0.1 \mathrm{mM}$
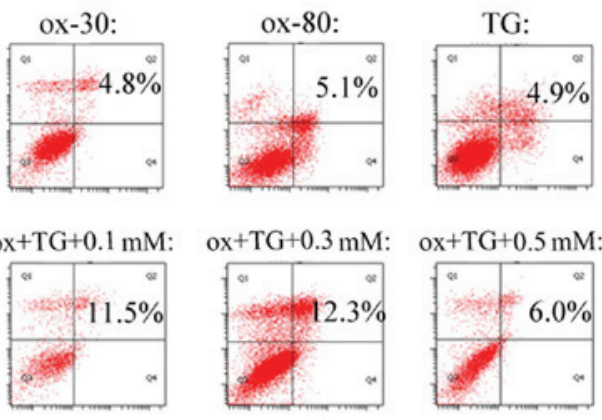

B
$0.5 \mathrm{mM}+\mathrm{TG}:$

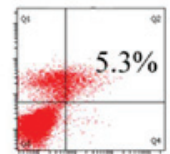

$0.5 \mathrm{mM}+\mathrm{ox}+\mathrm{TG}$ :

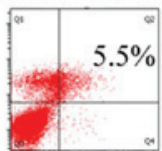

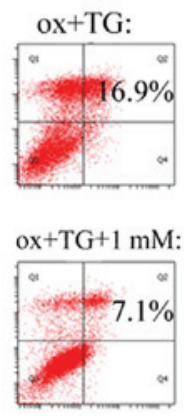

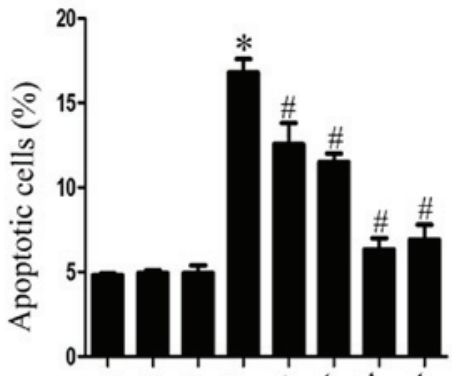

$0 \times 30,80 \times 0 \times 0$
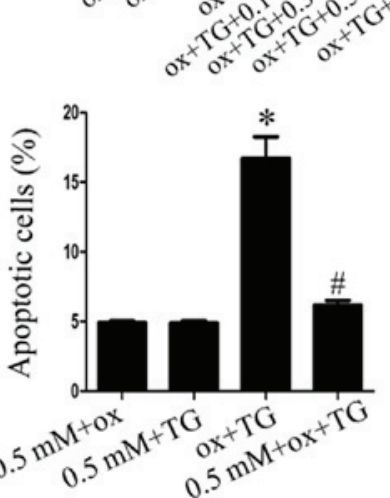

Figure 1. Metformin suppressed oxidized low density lipoprotein (ox-LDL) loaded-macrophage apoptosis. (A) ox-LDL ( 30 or $80 \mu \mathrm{g} / \mathrm{ml}$ ) or thapsigargin (TG) stimulation alone did not induce macrophage apoptosis. However, ox-LDL $(80 \mu \mathrm{g} / \mathrm{ml})$ and TG treatment together induced prominent macrophage apoptosis ( $\mathrm{P}<0.05$ vs. TG treatment), while metformin could reverse this effect with indicated concentration (from 0.1 to $1 \mathrm{mM}$; ${ }^{~} \mathrm{P}<0.05$ vs. ox+TG). The data presented here are the mean \pm standard deviation (SD) of three independent experiments. (B) Pretreating macrophages with metformin $(0.5 \mathrm{mM})$ for $24 \mathrm{~h}$ could suppress ox-LDL and TG induced apoptosis ( ${ }^{\#} \mathrm{P}<0.05 \mathrm{vs}$. ox $\left.+\mathrm{TG}\right)$. The data presented here are the mean $\pm \mathrm{SD}$ of three independent experiments.
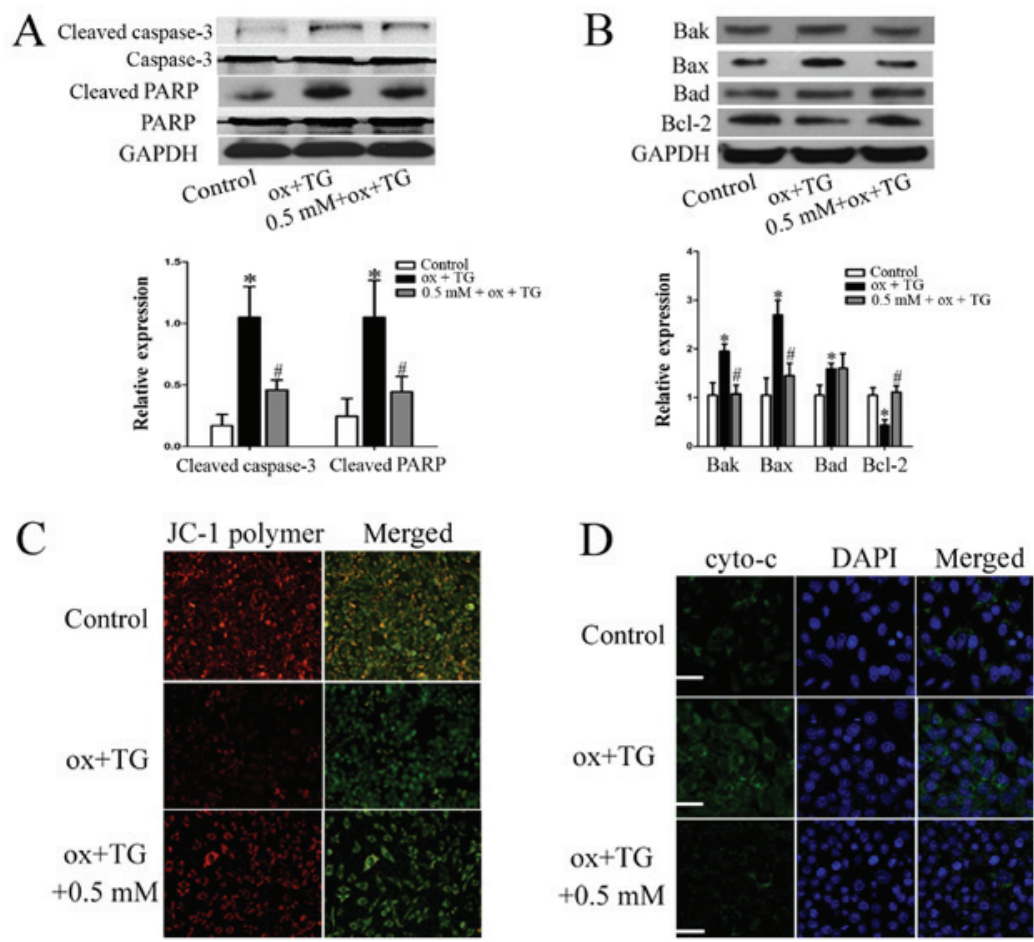

Figure 2. Metformin inhibited oxidized low density lipoprotein (ox-LDL)-induced caspase-3 clevage mitochondrial membrane potential depolarization and cytochrome $c$ (cyto-c) release. (A) ox-LDL and thapsigargin (TG) stimulation promoted PARP cleavage, which is a marker of apoptosis, but together with metformin treatment attenuated it $\left({ }^{*} \mathrm{P}<0.05\right.$ vs. control; " $\mathrm{P}<0.05$ vs. ox $\left.+\mathrm{TG}\right)$. The data presented here are the mean \pm standard deviation (SD) of three independent experiments. (B) ox-LDL and TG stimulation induced expression of the pro-apoptotic proteins Bax and Bak and suppressed anti-apoptotic protein Bcl-2, but metformin inhibited Bax and Bak and promotes Bad and Bcl- $2\left({ }^{*} \mathrm{P}<0.05\right.$ vs. control; ${ }^{*} \mathrm{P}<0.05$ vs. ox $\left.+\mathrm{TG}\right)$. The data presented here are the mean \pm SD of three independent experiments. (C) ox-LDL and TG treatment induced mitochondrial membrane potential depolarization in macrophages, while metformin reversed this effect. Green and red fluorescence indicate depolarized and polarized mitochondrial membrane potentials, respectively. Scale bars: $80 \mu \mathrm{m}$. (D) ox-LDL and TG treatment promotes cyto-c release (Green) from mitochondrion, but metformin treatment reduced it. Blue fluorescence represents cell nucleus. Scale bars: $50 \mu \mathrm{m}$. 

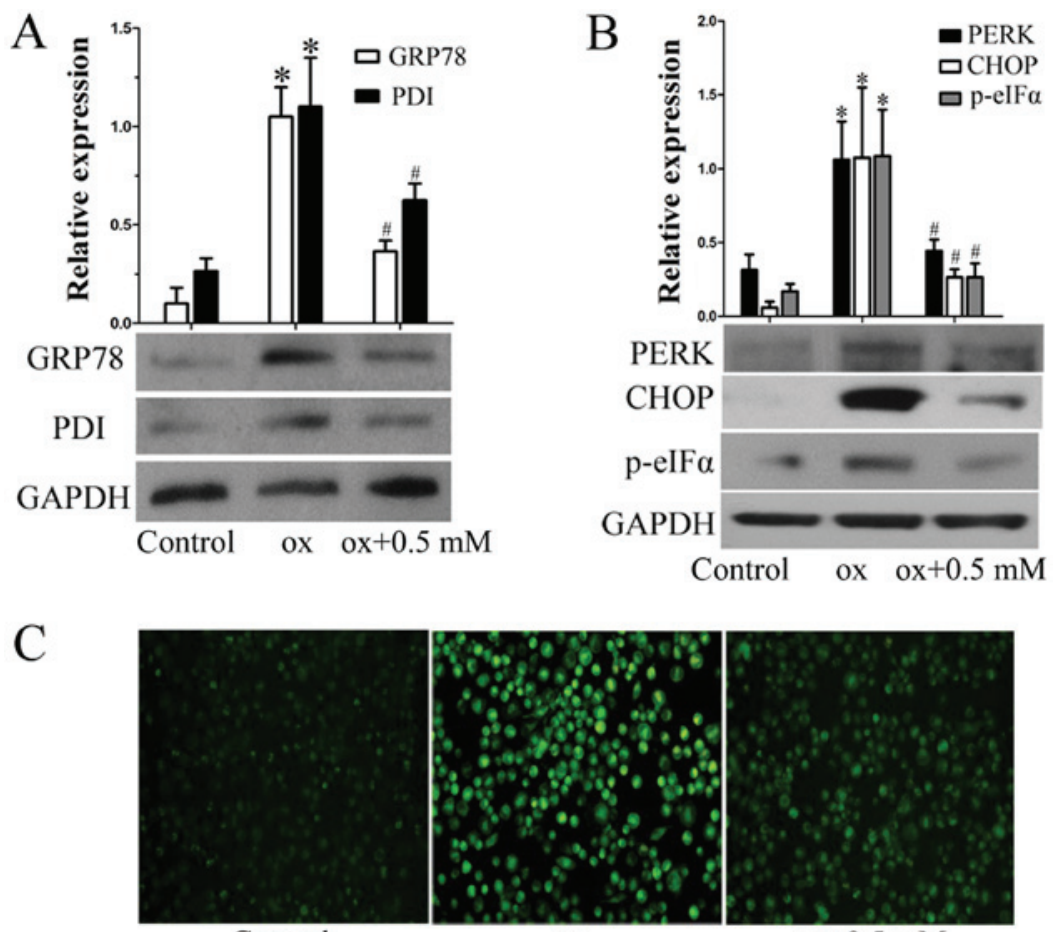

Control

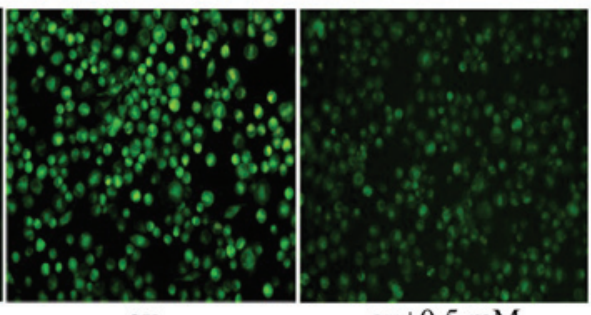

ox

$\mathrm{ox}+0.5 \mathrm{mM}$

Figure 3. Metformin attenuated oxidized low density lipoprotein (ox-LDL)-induced endoplasmic reticulum stress and ROS generation in macrophage. (A) ox-LDL increased glucose-regulated protein 78 (GRP78) and protein disulfide isomerase (PDI) expression, which indicate the activation of endoplasmic reticulum stress. However, metformin treatment together inhibited them ( ${ }^{*} \mathrm{P}<0.05$ vs. control; ${ }^{*} \mathrm{P}<0.05$ vs. ox $)$. The data presented here are the mean \pm standard deviation (SD) of three independent experiments. (B) ox-LDL increased the expression of PERK, CHOP, and p-eIF $\alpha$, three related pathways activated during endoplasmic reticulum stress, which can also be reversed by metformin ( ${ }^{*} \mathrm{P}<0.05$ vs. control; ${ }^{*} \mathrm{P}<0.05$ vs. ox). The data presented here are the mean $\pm \mathrm{SD}$ of three independent experiments. (C) ox-LDL treatment for $24 \mathrm{~h}$ promotes ROS generation (green) while metformin reduced it. Scale bars: $100 \mu \mathrm{m}$.

decreased when macrophages were treated with ox-LDL and TG. However, metformin reversed the upregulation of Bak and Bax and the down-regulation of Bcl-2 (Fig. 2B). Next, we also detected mitochondrial membrane potential alteration and cyto-c distribution. As shown in Fig. 1, metformin alleviated ox-LDL-induced mitochondrial membrane depolarization (Fig. 2C) and cyto-c release (Fig. 2D). Taken together, these results implicated that metformin suppresses mitochondrial pathway-mediated macrophage apoptosis triggered by ox-LDL and TG.

Metformin attenuate sox-LDL-induced ER stress and ROS generation in macrophages. Endoplasmic reticulum stress triggered by ox-LDL contributed to ROS generation and subsequent macrophage apoptosis, which had been reported by Tracie A (16). Whether metformin influenced ox-LDL-induced ER stress and subsequent ROS generation was unknown. The upregulation of ER chaperones, including GRP78 and protein disulfide isomerase (PDI), is a pivotal marker of ER stress. As shown in Fig. 3A, both GRP78 and PDI were induced by ox-LDL, while metformin partly canceled this promotion. In ER stress events, PERK separates from GRP78 deactivated eIF2 $\alpha$ through phosphorylation, which terminates protein synthesis and subsequently activates the downstream proapoptotic protein CHOP $(17,18)$. This phenomenon was also detected in ox-LDL-treated macrophages (Fig. 3). Metformin addition simultaneously reversed the ox-LDL induced upregulation of PERK, p-eIF2 $\alpha$, and CHOP (Fig. 3B). Moreover, ROS generation was also suppressed by metformin under ox-LDL treatment
(Fig. 3C). In conclusion, metformin can inhibit ox-LDL-induced ER stress and ROS generation, leading to the decrease of macrophage apoptosis.

Metformin inhibits lipid uptake through reducing scavenger receptor expression. Lipid accumulation continually in macrophages contributes to several biological disorders, including ER stress and mitochondrial dysfunction, resulting in apoptosis $(19,20)$. Consistent with Song's study (21), pretreatment of macrophages with metformin for $24 \mathrm{~h}$ suppressed lipid uptake (Fig. 4A). Scavenger receptors, including SRA, CD36, and LOX-1, had been reported to mediate lipid uptake, primarily in macrophages $(22,23)$. As shown in Fig. 4B and C, metformin treatment decreased ox-LDL-induced CD36 and SRA expression but had no significant effect on LOX-1 expression. Above all, metformin attenuated lipid uptake by inhibiting the expression of CD36 and SRA.

Metformin inhibits CD36 and SRA expression through suppressing $\beta$-catenin and $A P-1$ respectively. In our previous study, we identified that $\beta$-catenin increased CD36 transcription through cooperating with PPAR- $\gamma$ in macrophages (24). Thus, we investigated whether decreased expression of CD36 under metformin treatment was caused by the inhibition of $\beta$-catenin and PPAR- $\gamma$. To test our hypothesis, we tested the total and nuclear content of $\beta$-catenin and PPAR- $\gamma$ respectively. As shown in Fig. 5A, metformin indeed suppressed ox-LDL-induced $\beta$-catenin and PPAR- $\gamma$ upregulation. Simultaneously, nuclear content of $\beta$-catenin and PPAR- $\gamma$ 


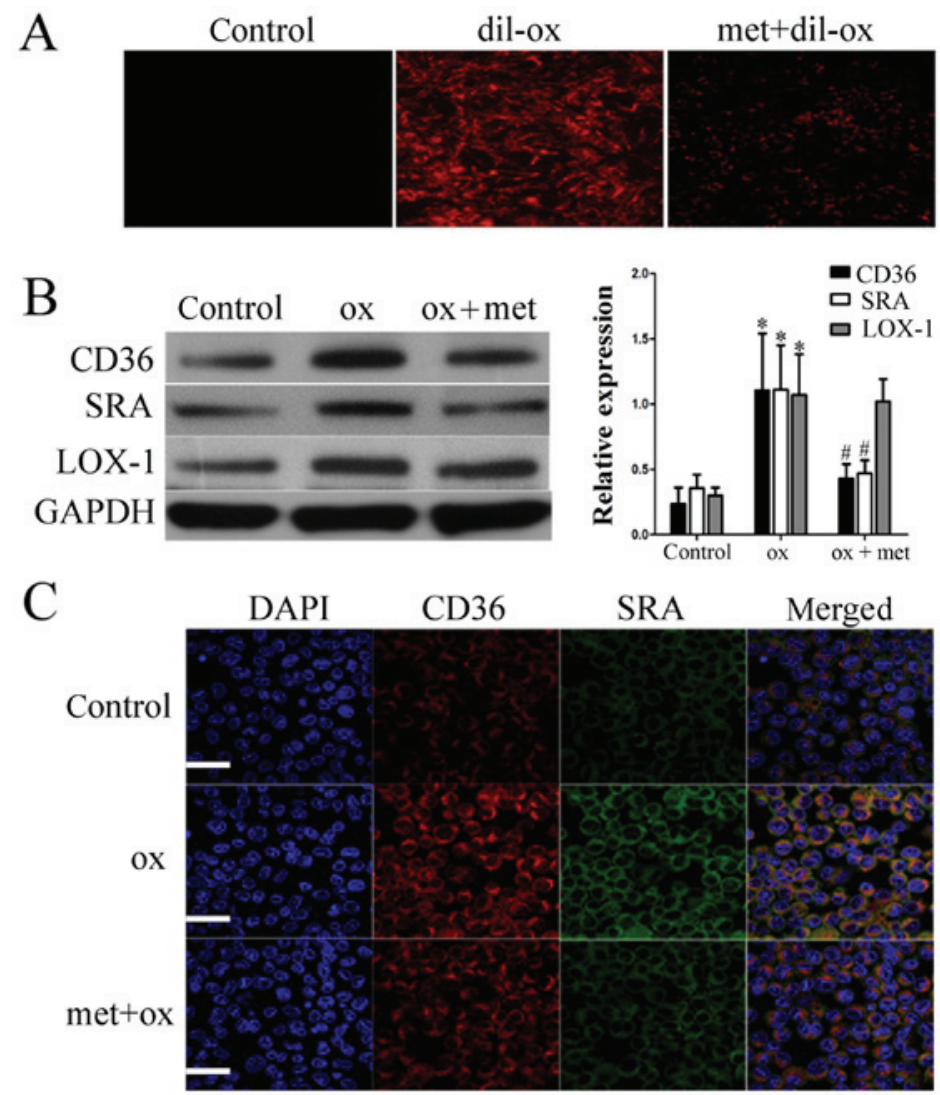

Figure 4. Metformin inhibited lipid uptake through downregulating scavenger receptors. (A) Macrophages were pretreated with metformin for $24 \mathrm{~h}$, and then stimulated with dil-oxidized low density lipoprotein (ox-LDL) for $30 \mathrm{~min}$. Fluorescence microscope imaging of dil-ox-LDL (red) is shown. Scale bars: $50 \mu \mathrm{m}$. (B) ox-LDL treatment for $24 \mathrm{~h}$ increased the expression of CD36, SRA, and LOX-1, but metformin stimulation inhibited the expression of CD36 and SRA $\left({ }^{*} \mathrm{P}<0.05\right.$ vs. control; ${ }^{*} \mathrm{P}<0.05$ vs. ox). The data presented here are the mean \pm standard deviation of three independent experiments. (C) Macrophages were treated with ox-LDL or ox-LDL and metformin together for $24 \mathrm{~h}$. Fluorescence microscope imaging of CD36 (red) and SRA (green) are shown. Scale bars: $60 \mu \mathrm{m}$.
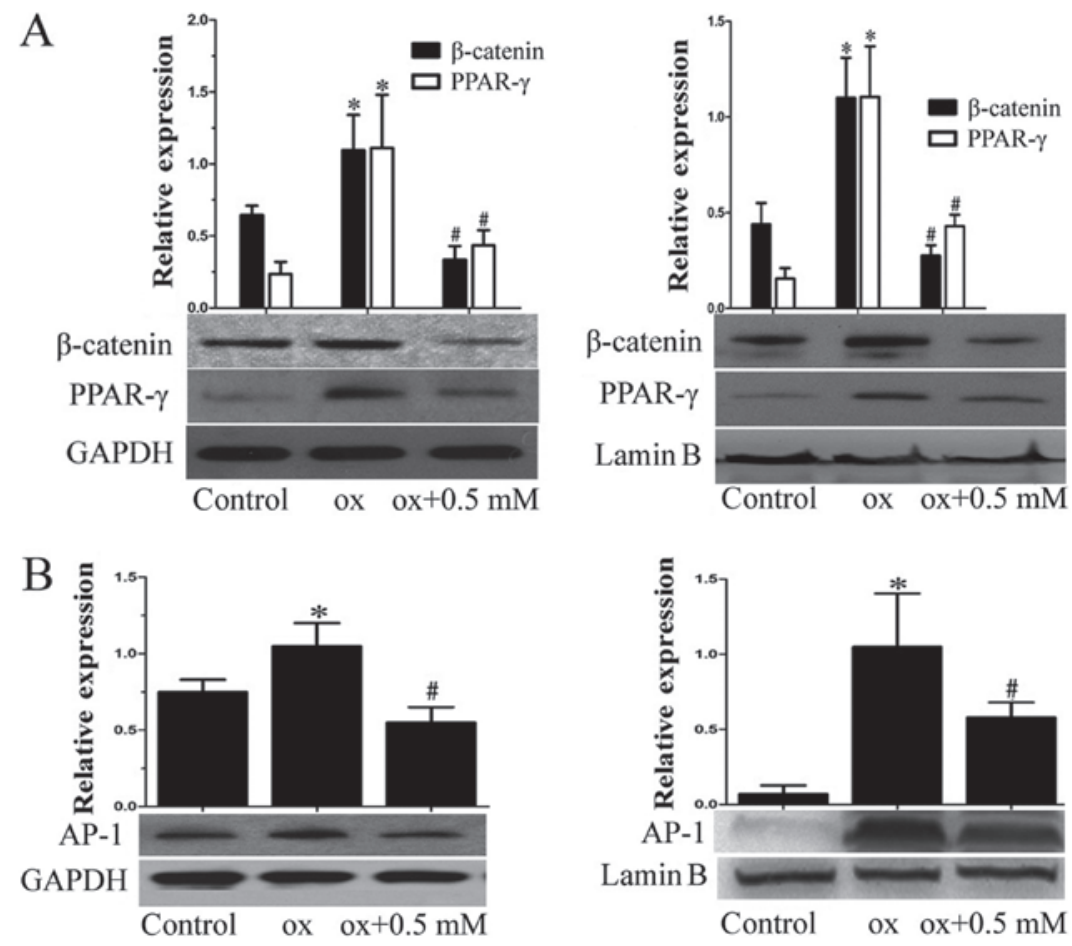

Figure 5. Metformin inhibited CD36 and SRA expression through suppressing beta-catenin nuclear expression, Wnt pathway, and AP-1 respectively. (A) Oxidized low density lipoprotein (ox-LDL) treatment upregulated total protein levels of $\beta$-catenin and PPAR- $\gamma$, while metformin $(0.5 \mathrm{mM})$ suppressed it. Nuclear expression of $\beta$-catenin and PPAR- $\gamma$ were increased following ox-LDL stimulation but metformin reversed it ( $\mathrm{P}<0.05$ vs. control; ${ }^{*} \mathrm{P}<0.05$ vs. ox). The data presented here are the mean \pm standard deviation (SD) of three independent experiments. (B) ox-LDL increased total or nuclear levels of AP-1 expression but metformin inhibited it in macrophages ( $\mathrm{P}<0.05$ vs. control; ${ }^{*} \mathrm{P}<0.05$ vs. ox). The data presented here are the mean $\pm \mathrm{SD}$ of three independent experiments. 
induced by ox-LDL was also inhibited by metformin. Previous studies had demonstrated that activating protein-1 (AP-1) binding element located between -67 and -50 bp relative to the transcriptional start site was critical for macrophage SRA activity $(25,26)$. Therefore, we speculated whether metformin inhibited SRA expression through controlling AP-1 expression. As shown in Fig. 5B, the increased expression of total or nuclear AP-1 stimulated by ox-LDL in macrophages was indeed partly canceled by metformin. Thus, we concluded that metformin inhibited CD36 and SRA expression through suppressing $\beta$-catenin and PPAR- $\gamma$ and AP-1 expression, respectively.

\section{Discussion}

In recent years, many studies have found that metformin has many excess effects on blood vessels besides its hypoglycemic effect (27-29). Clinical researchers have also identified that metformin was the only hypoglycemic drug which could lower the risk of cardio-cerebrovascular complications of T2DM patients $(30,31)$. Regarding the relationship between metformin and atherosclerosis, studies determined that metformin could suppress diabetes-related atherosclerosis via inhibiting Drpl-mediated mitochondrial fission (32). Furthermore, metformin could ameliorate the pro-inflammatory state in patients with carotid artery atherosclerosis (33). With the deepening of the research on metformin, many studies have identified its anti-inflammation effects. Song et al observed that metformin reduced lipid accumulation in macrophages by inhibiting FOXO1-mediated transcription of fatty acid-binding protein 4 (21). Koren-Gluzer et al demonstrated that metformin inhibits macrophage cholesterol biosynthesis rate through attenuated oxidative stress (34). In addition, metformin could inhibit monocyte-to-macrophage differentiation via AMPK-mediated inhibition of STAT3 activation, which may play an important role in atherosclerosis (35). Since macrophages play a vital role in the progress of atherosclerosis, we assume that metformin attenuated atherosclerosis may be related to the inhibition of lipid uptake in macrophages.

In this study, we further investigate the mechanism underlying the inhibition of lipid uptake in macrophages by metformin. By detecting scavenger receptor expression, we found that metformin treatment suppressed CD36 and SRA expression in macrophages with ox-LDL. Combined with previous works, canonical WNT pathway and AP-1 were observed to be involved in metformin-mediated reduction of CD36 and SRA in macrophages with ox-LDL stimulation $(24,26)$. Since several studies had confirmed the beneficial effect of metformin on slowing atherosclerosis development $(36,37)$, our study is the first to demonstrate the detailed mechanism involved in atherosclerosis alleviation by metformin, which provides the theoretical basis for applying metformin for atherosclerosis treatment. In summary, we identified that metformin could suppress ox-LDL loaded-macrophage apoptosis by alleviating mitochondrial membrane depolarization, cyto-c release, ER stress and ROS generation. Additionally, metformin could inhibit lipid uptake through down-regulation of SRA and CD36 by suppressing $\beta$-catenin and NF-kB.

\section{References}

1. Hansson GK and Hermansson A: The immune system in atherosclerosis. Nat Immunol 12: 204-212, 2011

2. Yan H, Wang S, Li Z, Zhao W, Wang Z, Sun Z, Pan Y and Zhu J: Upregulation of miRNA-155 expression by OxLDL in dendritic cells involves JAK1/2 kinase and transcription factors YY1 and MYB. Int J Mol Med 37: 1371-1378, 2016.

3. Moore KJ and Tabas I: Macrophages in the pathogenesis of atherosclerosis. Cell 145: 341-355, 2011.

4. Libby P, Ridker PM and Hansson GK: Progress and challenges in translating the biology of atherosclerosis. Nature 473: 317-325, 2011.

5. Bailey CJ and Turner RC: Metformin. N Engl J Med 334: 574-579, 1996.

6. Hundal RS, Krssak M, Dufour S, Laurent D, Lebon V, Chandramouli V, Inzucchi SE, Schumann WC, Petersen KF, Landau BR and Shulman GI: Mechanism by which metformin reduces glucose production in type 2 diabetes. Diabetes 49: 2063-2069, 2000.

7. Hundal RS and Inzucchi SE. Metformin-New understandings, new uses. Drugs 63: 1879-1894, 2003.

8. Fitch K, Abbara S, Lee H, Stavrou E, Sacks R, Michel T, Hemphill L, Torriani M and Grinspoon S: Effects of lifestyle modification and metformin on atherosclerotic indices among HIV-infected patients with the metabolic syndrome. AIDS 26: 587-597, 2012.

9. Song J, Ren PP, Zhang L, Wang XL, Chen L and Shen YH: Metformin reduces lipid accumulation in macrophages by inhibiting FOXO1-mediated transcription of fatty acid-binding protein 4. Biochem Biophys Res Commun 393: 89-94, 2010.

10. Koren-Gluzer M, Aviram M and Hayek T: Metformin inhibits macrophage cholesterol biosynthesis rate: Possible role for metformin-induced oxidative stress. Biochem Biophys Res Commun 439: 396-400, 2013.

11. Daigneault M, Preston JA, Marriott HM, Whyte MK and Dockrell DH: The identification of markers of macrophage differentiation in PMA-stimulated THP-1 cells and monocyte-derived macrophages. PLoS One 5: e8668, 2010.

12. Wang Q, Ji J, Hao S, Zhang M, Li K and Qiao T: Iron together with lipid downregulates protein levels of ceruloplasmin in macrophages associated with rapid foam cell formation. J Atheroscler Thromb 23: 1201-1211, 2016.

13. Yan H, Li Y, Peng X, Huang D, Gui L and Huang B: Resistance of mitochondrial DNA-depleted cells against oxidized low-density lipoprotein-induced macrophage pyroptosis. Mol Med Rep 13: 4393-4399, 2016

14. Goeritzer M, Schlager S, Radovic B, Madreiter CT, Rainer S, Thomas G, Lord CC, Sacks J, Brown AL, Vujic N, et al: Deletion of CGI-58 or adipose triglyceride lipase differently affects macrophage function and atherosclerosis. J Lipid Res 55: 2562-2575, 2014.

15. Aflaki E, Doddapattar P, Radović B, Povoden S, Kolb D, Vujić N, Wegscheider M, Koefeler H, Hornemann T, Graier WF, et al: C16 ceramide is crucial for triacylglycerol-induced apoptosis in macrophages. Cell Death Dis 3: e280, 2012.

16. Seimon TA, Nadolski MJ,Liao X, Magallon J, Nguyen M, Feric NT, Koschinsky ML, Harkewicz R, Witztum JL, Tsimikas S, et al: Atherogenic lipids and lipoproteins trigger CD36-TLR2-dependent apoptosis in macrophages undergoing endoplasmic reticulum stress. Cell Metab 12: 467-482, 2010.

17. Cnop M, Igoillo-Esteve M, Cunha DA, Ladrière L and Eizirik DL: An update on lipotoxic endoplasmic reticulum stress in pancreatic beta-cells. Biochem Soc Trans 36: 909-915, 2008.

18. Ron D and Walter P: Signal integration in the endoplasmic reticulum unfolded protein response. Nat Rev Mol Cell Biol 8: 519-529, 2007.

19. Yao S, Zong C, Zhang Y, Sang H, Yang M, Jiao P, Fang Y, Yang N, Song G and Qin S: Activating transcription factor 6 mediates oxidized LDL-induced cholesterol accumulation and apoptosis in macrophages by up-regulating CHOP expression. J Atheroscler Thromb 20: 94-107, 2013.

20. Sekiya M, Yamamuro D, Ohshiro T, Honda A, Takahashi M, Kumagai M, Sakai K, Nagashima S, Tomoda H, Igarashi M, et al: Absence of Nceh1 augments 25-hydroxycholesterol-induced ER stress and apoptosis in macrophages. J Lipid Res 55: 2082-2092, 2014.

21. Song J, Ren P, Zhang L, Wang XL, Chen L and Shen YH: Metformin reduces lipid accumulation in macrophages by inhibiting FOXO1-mediated transcription of fatty acid-binding protein 4. Biochem Biophys Res Commun 393: 89-94, 2010. 
22. Murphy JE, Tedbury PR, Homer-Vanniasinkam S, Walker JH and Ponnambalam S: Biochemistry and cell biology of mammalian scavenger receptors. Atherosclerosis 182: 1-15, 2005.

23. Yan H, Wang S, Li Z, Sun Z, Zan J, Zhao W, Pan Y, Wang Z, Wu M and Zhu J: Rspo2 suppresses CD36-mediated apoptosis in oxidized low density lipoprotein-induced macrophages. Mol Med Rep 14: 2945-2952, 2016.

24. Wang S, Sun Z, Zhang X, Li Z, Wu M, Zhao W, Wang H, Chen T, Yan $\mathrm{H}$ and Zhu J: Wnt1 positively regulates CD36 expression via TCF4 and PPAR- $\gamma$ in macrophages. Cell Physiol Biochem 35: 1289-1302, 2015

25. Zhao W, Sun Z, Wang S, Li Z and Zheng L: Wnt1 participates in inflammation induced by lipopolysaccharide through upregulating scavenger receptor A and NF-kB. Inflammation 38: 1700-1706, 2015.

26. Mietus-Snyder M, Glass CK and Pitas RE: Transcriptional activation of scavenger receptor expression in human smooth muscle cells requires AP-1/c-Jun and C/EBPbeta: Both AP-1 binding and JNK activation are induced by phorbol esters and oxidative stress. Arterioscler Thromb Vasc Biol 18: 1440-1449, 1998.

27. Gillessen S, Gilson C, James N, Adler A, Sydes MR and Clarke N; STAMPEDE Trial Management Group: Repurposing metformin as therapy for prostate cancer within the STAMPEDE trial platform. Eur Urol 70: 906-908, 2016.

28. Cameron AR, Morrison VL, Levin D, Mohan M, Forteath C, Beall C, McNeilly AD, Balfour DJ, Savinko T, Wong AK, et al: Anti-inflammatory effects of metformin irrespective of diabetes status. Circ Res 119: 652-665, 2016.

29. Ramakrishna G, Sen B, Trehanpati N and Sarin SK: Repurposing of metformin in liver injury: The JNK conundrum. J Hepatol 64: 749-750, 2016.

30. Gerstein HC, Miller ME, Ismail-Beigi F, Largay J, McDonald C, Lochnan HA and Booth GL; ACCORD Study Group: Effects of intensive glycaemic control on ischaemic heart disease: Analysis of data from the randomised, controlled ACCORD trial. Lancet 384: 1936-1941, 2014.
31. ACCORD Study Group: Nine-year effects of 3.7 years of intensive glycemic control on cardiovascular outcomes. Diabetes Care 39: 701-708, 2016.

32. Wang Q, Zhang M, Torres G, Wu S, Ouyang C, Xie Z and Zou MH: Metformin suppresses diabetes-accelerated atherosclerosis via the inhibition of Drpl-mediated mitochondrial fission. Diabetes 66: 193-205, 2017.

33. Xu W, Deng YY, Yang L, Zhao S, Liu J, Zhao Z, Wang L, Maharjan P, Gao S, Tian Y, et al: Metformin ameliorates the proinflammatory state in patients with carotid artery atherosclerosis through sirtuin 1 induction. Transl Res 166: 451-458, 2015.

34. Koren-Gluzer M, Aviram M and Hayek T: Metformin inhibits macrophage cholesterol biosynthesis rate: Possible role for metformin-induced oxidative stress. Biochem Biophys Res Commun 439: 396-400, 2013.

35. Vasamsetti SB, Karnewar S, Kanugula AK, Thatipalli AR, Kumar JM and Kotamraju S: Metformin inhibits monocyte-to-macrophage differentiation via AMPK-mediated inhibition of STAT3 activation: Potential role in atherosclerosis. Diabetes 64: 2028-2041, 2015.

36. Liu X, Mei T, Chen W and Ye S: Comparison of antidiabetic medications during the treatment ofatherosclerosis in T2DM patients. Mediators Inflamm 2017: 5032708, 2017.

37. Luo F, Guo Y, Ruan GY, Long JK, Zheng XL, Xia Q, Zhao SP, Peng DQ, Fang ZF and Li XP: Combined use of metformin and atorvastatin attenuates atherosclerosis in rabbits fed a high-cholesterol diet. Sci Rep 7: 2169, 2017.

cC) (1) $\odot$ This work is licensed under a Creative Commons cc) $\mathrm{EY}$ NC ND Attribution-NonCommercial-NoDerivatives 4.0 International (CC BY-NC-ND 4.0) License. 